\title{
Different mutational characteristics of the subsets of EGFR-tyrosine kinase inhibitor sensitizing mutation-positive lung adenocarcinoma
}

\author{
Eun Young Kim, Arum Kim', Gaeun Lee ${ }^{2}$, Hangsuck Lee ${ }^{2}$ and Yoon Soo Chang ${ }^{3^{*}}$
}

\begin{abstract}
Background: A subset of lung adenocarcinoma with EGFR-tyrosine kinase inhibitor sensitizing mutations (mEGFR) is common in non-smokers and women, suggesting that mutational stressors other than smoking are involved.

Methods: Targeted sequencing using a custom panel containing 70 cancer-related genes were performed from 73 cases of lung adenocarcinoma with mEGFR (study cohort). In parallel, publicly available data of 47 TCGA-LUAD cases with mEGFR (LUAD cohort) were extracted from the GDC data portal and analyzed by non-negative matrix factorization using the Maftools package.

Results: In the study cohort, the C > A transversions accounted for $12.9 \%$ of all single nucleotide variations (SNVs), comprising the second smallest proportion among SNVs. The E19del-subgroup had a significantly lower mutational burden with significantly higher Ti/TV ratio than the SNV-subgroup, which includes cases with L858R and other EGFRTKI sensitizing SNVs. ( $P=0.0326$ and 0.0002 , respectively, Mann-Whitney $U$ test). In the LUAD cohort, the mutational burden was substantially lower than in other TCGA cancer cohorts, and the frequency of C > A transversions was 30 . 3\%, occupying the second frequency. The E19del-subgroup had a lower mutational burden overall and a higher Ti/TV ratio than the SNV-subgroup ( $P=0.0497$ and $P=0.0055$, respectively, Mann-Whitney $\mathrm{U}$ test). Smoking-related signature 4 was observed only in the L858R-subgroup, while ignature 30 and 5 was observed in both groups.

Conclusions: Lung adenocarcinoma with mEGFR(+) has a lower mutational burden and does not show a characteristic mutation pattern influenced by smoking. E19del and L858R, which are representative subtypes of mEGFR(+) lung adenocarcinoma, differ in terms of mutational spectrum, as the E19del-subgroup has a lower mutation burden and a higher Ti/Tv ratio than the SNV-subgroup. These findings could help explain the differences in the responses to EGFR-TKIs and in the clinical courses between the two lung adenocarcinoma subgroups.
\end{abstract}

Keywords: EGFR, Lung adenocarcinoma, Mutational signatures, Next-generation sequencing, Sensitizing mutation, Tyrosine kinase inhibitor

\footnotetext{
* Correspondence: yschang@yuhs.ac

${ }^{3}$ Department of Internal Medicine, Yonsei Univeristy College of Medicine, 4th

Floor Research Center for Future Medicine, 63-Gil 20, Eonju-ro, Gangnam-gu,

Seoul, Republic of Korea

Full list of author information is available at the end of the article
}

(c) The Author(s). 2018 Open Access This article is distributed under the terms of the Creative Commons Attribution 4.0 International License (http://creativecommons.org/licenses/by/4.0/), which permits unrestricted use, distribution, and reproduction in any medium, provided you give appropriate credit to the original author(s) and the source, provide a link to the Creative Commons license, and indicate if changes were made. The Creative Commons Public Domain Dedication waiver (http://creativecommons.org/publicdomain/zero/1.0/) applies to the data made available in this article, unless otherwise stated. 


\section{Background}

According to the 2015 annual report, 1,824,700 new lung cancer cases are diagnosed each year, which accounts for $13 \%$ of all cancers, excluding non-melanoma skin cancers. In addition, it is still the leading cause of cancer mortality worldwide, suggesting that lung cancer is a major problem for healthcare worldwide [1]. According to the 2015 yearbook of the National Cancer Registration and Statistics in Korea, lung cancer occurred in 66.0 per 100,000 males and 28.7 per 100,000 females [2]. Compared to other countries, Korea is 10th in the incidence of male lung cancer and 4th in the incidence of female lung cancer [3]. The total incidences in Korea are not significantly different from other countries, but the incidence of lung cancer is higher in non-smokers and women in Korea, and epidermal growth factor receptor (EGFR) mutations are detected much more frequently than in Western countries.

The main causes of lung cancer are direct and indirect smoking, radon, indoor emissions from household combustion, and exhaust from diesel engines (https:// monographs.iarc.fr/agents-classified-by-the-iarc/) [4]. Mutational analysis of lung cancer using publicly available data such as TCGA has shown that the smoke-related signature, with many $\mathrm{C}>\mathrm{A}$ transversions, is a dominant signature in lung adenocarcinoma and lung squamous cell carcinoma [5]. Somatic mutations in cancer are caused infidelity of the DNA replication machinery as well as and defects in DNA repair mechanisms following exposure to endogenous or exogenous mutagens [6]. The somatic mutations observed in some cancers are significantly related to exposure to a specific carcinogen, such as smoking in lung cancer and ultraviolet light in skin cancer [7].

Certain mutational processes in cancer often accompany unique combinations of mutation types called signatures $[8,9]$. Recently, Alexandrov et al. developed a theoretical model and computational framework that could deconstruct unique patterns of somatic mutations using cancer specimen sequencing data based on the analysis of somatic substitutions obtained from whole genome sequencing of breast cancer patients $[10,11]$. Among the 30 signatures, signature 4 , which is characterized by a majority of $\mathrm{C}>\mathrm{A}$ mutations along with some other base substitution classes, is found only in cancer types in which smoking is a major risk factor and in epithelial cancers that are directly exposed to cigarette smoke. The mutational signature is similar to the mutational pattern resulting from exposing cells to benzo [a] pyrene, a major carcinogen in tobacco. This mutational pattern occurs in the process of nucleotide excisional repair after binding of a bulky DNA adduct to the guanine [5, 8].

Given that lung adenocarcinoma with EGFR-tyrosine kinase inhibitor (TKI) sensitizing mutation (mEGFR) is common in light and/or non-smokers, Asians, and women, it is expected to have mutational pressures other than cigarette smoking, [12]. The L858R mutation and the exon 19 deletion (E19del), which includes the LREA motif, comprise up to $90 \%$ of EGFR mutations, followed by L861Q, G719X, and rare mutations [13]. The clinical outcomes of mEGFR positive lung cancers have dramatically improved due to the development of target drugs, but these cancers eventually acquire drug resistance and show disease progression $[14,15]$. The clinical courses and responses to EGFR-TKIs differ between the E19del and L858R groups, which are representative subtypes of mEGFR [16]. Therefore, the identification of carcinogenesis by the estimation of significant mutagenic stressors is needed, along with a proactive approach for these subtypes of cancer.

Therefore, we investigated whether there is a distinctive mutation pattern in the subtypes of lung adenocarcinoma responsive to EGFR-TKI. Targeted sequencing was performed on major cancer-related genes using lung adenocarcinoma with mEGFR (study cohort), and the characteristics of the obtained mutations were analyzed. In addition, the mutation characteristics of the L858R and E19del subtypes, which occupy the majority of the mEGFR, were compared and analyzed. Finally, whole exome sequencing data from TCGA-LUAD with mEGFR (LUAD cohort), which are publicly available, were analyzed and used to verify the mutational characteristics of study cohort. The characteristics of the genetic variations were analyzed in the context of the mutational signature proposed by Alexandrov et al. [17].

\section{Methods}

\section{Study cases}

A total of 74 lung adenocarcinoma tissues which met the following criteria were randomly selected from the tissue archives of two affiliated hospitals, Severance Hospital and Gangnam Severance Hospital, of Yonsei Medical Center (study cohort). Each specimen (1) is a pathologically confirmed lung adenocarcinoma, (2) had history of curative surgical resection, (3) has a submission of informed consent for sequencing of major cancer related genes, (4) was confirmed to have mEGFR either by Sanger sequencing or PNA clamping methods, and (5) has submission of informed consent for tissue collection. This study was approved by the IRB of Gangnam Severance Hospital (IRB \#3-2017-0059) and was carried out in compliance with the Declaration of Helsinki (https://www.wma.net/policies-post/wma-declaration-ofhelsinki-ethical-principles-for-medical-research-involving-human-subjects/) and Korean GCP guidelines. In order to further evaluate the mutational characteristics of lung adenocarcinoma with mEGFR, publicly available data were extracted from the GDC Data Portal of The Cancer Genome Atlas (TCGA https://portal.gdc.cancer.gov/). Among the 585 TCGA-LUAD cases, 81 cases harbor mutations in 
the EGFR gene (ENSG00000146648) with 47 types listed in TCGA-LUAD (Additional file 1: Table S1). A total of 7577 mutations were found in 48 TCGA-LUAD cases; TCGA-55-8506 has 2305 mutations alone, accounting for $30.4 \%$ of all mutations, and was excluded from further analysis. For this analysis, we selected 47 cases with 7 EGFR subtypes known as common mEGFR (LUAD cohort) (Additional file 2: Table S2 and Additional file 3: Table S3) (8).

Next generation sequencing: A $0.62 \mathrm{Mb}$ customized NGS panel containing 70 major cancer genes was constructed and sequenced using the Ion S5 NGS system (Thermo Fisher Scientific) (Additional file 4: Table S4). For the extraction of cancer-enriched gDNA, paraffin-embedded tissue samples were loaded onto silanated slides in $4-\mu \mathrm{m}$-thickness sections. Each slide was lightly stained with H\&E and examined for the presence of cancer cells. The cancer-enriched area as determined by an independent lung pathologist was marked and scraped with clean blades. gDNA was extracted using a QIAamp DNA FFPE Tissue Kit (Qiagen, Valencia, CA, USA). Fifty ng of extracted DNA was reacted with fragmentizer for 12 to 50 min (Archer) and the section of cut DNA was blunted and 5' phosphorylated with an end-repair enzyme (Archer). The end of the DNA was barcode-ligated through a reaction with an $\mathrm{MBC}$ adapter (Archer) for $15 \mathrm{~min}$, and 1st and 2nd PCR were performed using a primer set for the selected target genes. After measuring the prepared library with Qubit , $50 \mathrm{pmol}$ of sample was obtained and mixed with mineral oil to normalize it with beads. The sample was applied to a 540 Chip (Life Technology) and sequenced with the S5 sequencer (Life technology). The obtained results were analyzed by Archer Analysis 5.1 (Archer) and the median depth of the sequencing was 308X [164.5 738.5].

\section{Statistics}

Categorical and continuous variables were compared using $\chi^{2}$-tests and $t$-test, respectively. Differences in the distribution of continuous variables between the two independent samples and dependent samples were assessed by MannWhitney $U$ test and Wilcoxon signed-rank test, respectively. The Kruskal-Wallis test was used to compare the medians of three or more groups, and the Bonferroni method was used for the post-hoc test. The mutational signature was analyzed using $\mathrm{R}$ software and the Maftools package [18]. Briefly, the pattern of the mutation is analyzed under the 3-nucleotide context, which includes one nucleotide immediately $5^{\prime}$ and $3^{\prime}$ of the mutated base $[10,17]$ to characterize single base substitution using a 96-mutation classification by combining 6 substitution types and the $5^{\prime}$ and $3^{\prime}$ adjacent bases to the mutated base. The mutation patterns found in cancers are analyzed by non-negative matrix factorization (NMF), and compared to 30 types of mutational signatures [8]. All analyses were performed using SPSS version 23 (SPSS Inc., Chicago, IL, USA). All statistical tests were two-sided, and a P-value $<0.05$ was considered to be statistically significant.

\section{Results \\ Characteristics of study cohort}

The incidence of lung adenocarcinoma harboring EGFR-TKI-sensitizing mutations is higher in East Asia than in Western countries, and the proportion of non-smokers and women is high, suggesting the presence of mutagenic stressors other than cigarette smoking. We conducted targeted sequencing of 70 major cancer-related genes on a total 73 specimens recruited from 71 patients, including two cases with double primary cancer (Table 1 and Additional file 5: Table S5). These included 37 cases with EGFR exon19 deletion mutation, 35 cases with L858R mutation and one case with the L861Q mutation. The mean age of the patients was $60.34 \pm 10.01$ years, and they were comprised of 25 males and 46 females. Of these, 20 cases had a history of smoking, with a mean of $22.75 \pm 13.33$ pack-years.

\section{C > A transversion accounts for small fraction in study cohort}

The sequencing of 73 specimens revealed 530 mutations consisting of 490 single and double nucleotide substitutions, 1 insertion, and 39 deletions. The median of total mutations obtained from each sample was 6 [5 $~ 8]$ (Fig. 1A and B). These mutations were further classified according to the variant effect predictor (VEP) and the results are shown in Fig. $1 \mathrm{C}$ and Table 2 . $\mathrm{C}>\mathrm{A}$ transversion, which is attributed to cigarette smoking, is the most common type of SNV in lung cancer [5]; therefore, we questioned whether this finding also applies to Korean lung adenocarcinomas with mEGFR. Excluding the mutations which were assumed to be benign because they did not change protein behaviors by the VEP, and indels and doublets, a total of 363 SNVs were classified and analyzed (Fig. 1D and E). In this subset, $\mathrm{C}>\mathrm{T}$ transition accounted for the largest portion (27.3\%), followed by $\mathrm{C}>\mathrm{G}$ transition (20.4\%), whereas $\mathrm{C}>\mathrm{A}$ transversion occupied the second smallest fraction (12.9\%). The frequency of $\mathrm{C}>\mathrm{A}$ transversion was statistically significantly lower than those of other mutations, except for $\mathrm{T}>\mathrm{A}$ and $\mathrm{T}>\mathrm{G}$ transversions, which differs from the general notion that NSCLC harbors higher C $>$ A transversions due to cigarette smoking. The differences in the frequencies of $\mathrm{Ti}$ and $\mathrm{Tv}$ did not reach statistically significant level in each individual case (Fig. 1F).

\section{Lung adenocarcinoma with the E19del mutation has a significantly lower mutational burden, higher Ti/Tv ratio} To identify the presence of smoking effects, we examined whether there was a difference in the mutational 
Table 1 Demographic characteristics of the 2 cohorts according to the EGFR-TKI mutation subtypes

\begin{tabular}{|c|c|c|c|c|c|}
\hline & & & $\mathrm{SNV}^{*}$ & E19del ${ }^{* *}$ & P-value \\
\hline \multirow[t]{9}{*}{ Study cohort $(n=71)^{\dagger}$} & Age & & $62.58 \pm 9.67$ & $58.03 \pm 9.95$ & $0.0547^{\ddagger \neq}$ \\
\hline & Gender & Male & 14 & 11 & $0.6822^{\S}$ \\
\hline & & Female & 22 & 24 & \\
\hline & Smoking history & Ever smoker & 11 & 9 & $0.8497^{\S}$ \\
\hline & & None smoker & 25 & 26 & \\
\hline & Stage & । & 22 & 12 & $0.1100^{\S}$ \\
\hline & & $\|$ & 5 & 12 & \\
\hline & & III & 8 & 10 & \\
\hline & & IV & 1 & 1 & \\
\hline \multirow[t]{9}{*}{ LUAD cohort $(n=47) \S \S$} & Age & & $66.56 \pm 9.18$ & $65.81 \pm 10.40$ & $0.8138^{\ddagger \neq}$ \\
\hline & Gender & Male & 7 & 4 & $0.9234^{\S}$ \\
\hline & & Female & 10 & 14 & \\
\hline & Smoking history & Ever smoker & 15 & 4 & $0.0540^{\S}$ \\
\hline & & None smoker & 13 & 15 & \\
\hline & Stage & । & 13 & 7 & $0.9715^{\S}$ \\
\hline & & $\|$ & 7 & 5 & \\
\hline & & III & 6 & 5 & \\
\hline & & IV & 1 & 1 & \\
\hline
\end{tabular}

* Study cohort of SNV subgroup includes one L861Q variant and LUAD cohort of SNV subgroup includes 3 cases of L861Q, 2 G719A, and 1 G719C variant

** Includes E19 inframe del variants including LREA motif

${ }^{\dagger}$ Includes 2 cases with double primary tumor

\#\# P-values were obtained by independent sample t-test

$\S$ P-values were obtained by chi-square test

${ }^{\S \varsigma}$ The clinical information of 2 cases is not available

spectrum between cases with vs. without a smoking history. There was no statistically significant difference in the total number of mutations between smoker and non-smoker groups and between those with more than a 20-pack-year history and the other cases. There was no significant difference in the frequencies of $\mathrm{Ti}, \mathrm{Tv}$, and $\mathrm{Ti} / \mathrm{Tv}$ ratio according to the smoking history. These findings were similar to the comparison of mutation patterns according to gender, which showed no differences in the total mutational burden, frequencies of $\mathrm{Ti}$ and $\mathrm{Tv}$, and $\mathrm{Ti} / \mathrm{Tv}$ ratios between genders. Then, we questioned whether the subtypes of mEGFR are related to the difference in mutation burden, $\mathrm{Ti}, \mathrm{Tv}$, and $\mathrm{Ti} / \mathrm{Tv}$ ratio. In order to accomplish this, the cases were classified into either the mEGFR E19del-subgroup or the mEGFR SNV-subgroup, which includes cases with L858R, L861Q, and other EGFR-TKI sensitizing SNVs. In the E19del-subgroup, there was no statistically significant difference in the frequency of $\mathrm{Ti}$ and $\mathrm{Tv}$ in each individual, whereas the frequency of $\mathrm{Tv}$ was significantly higher than $\mathrm{Ti}$ in the mEGFR SNV-subgroup $(P=0.0020$, Wilcoxon signed rank test). There was a significant difference in the total number of mutations, as the E19del-subgroup had a lower mutational burden than the SNV-subgroup ( $P=0.0326$, Mann-Whitney U test).
The E19del-subgroup had lower Tv and a higher Ti and $\mathrm{Ti} / \mathrm{Tv}$ ratio than the SNV-subgroup $(P=0.0441,=0.0020$, and $=0.0002$, respectively, Mann-Whitney $U$ test, Fig. 2A D). As $C>A$ transversion was less frequent than in general lung cancer, this suggests that some mutagenic stress other than cigarette smoking likely exists in this subset of lung adenocarcinomas. To identify the mutagenic stressors that influence mEGFR-positive lung adenocarcinomas, we analyzed the mutational signature proposed by Alexandrov et al. (4). When the three best-matched mutational signatures were estimated using non-negative matrix factoralization (NMF), signature 5 and signature 3 was derived (Fig. 2E and F). Among smoking history, gender, and EGFR-mutation subtype, only EGFR subtype showed a difference in the distributions of $\mathrm{Ti}$ and $\mathrm{Tv}$. We further analyzed the mutational signatures between the E19del-subgroup and the SNV-subgroup using NMF. In the subgroup analysis on the mutational, signature 5 is the predominant mutational signature in this study cohort (Data not shown).

\section{LUAD cohort has a lower mutational burden than other TCGA cancer cohorts}

The mean age of the 47 selected cases was $66.20 \pm 9.44$ years, and the cases were comprised of 11 males, 34 


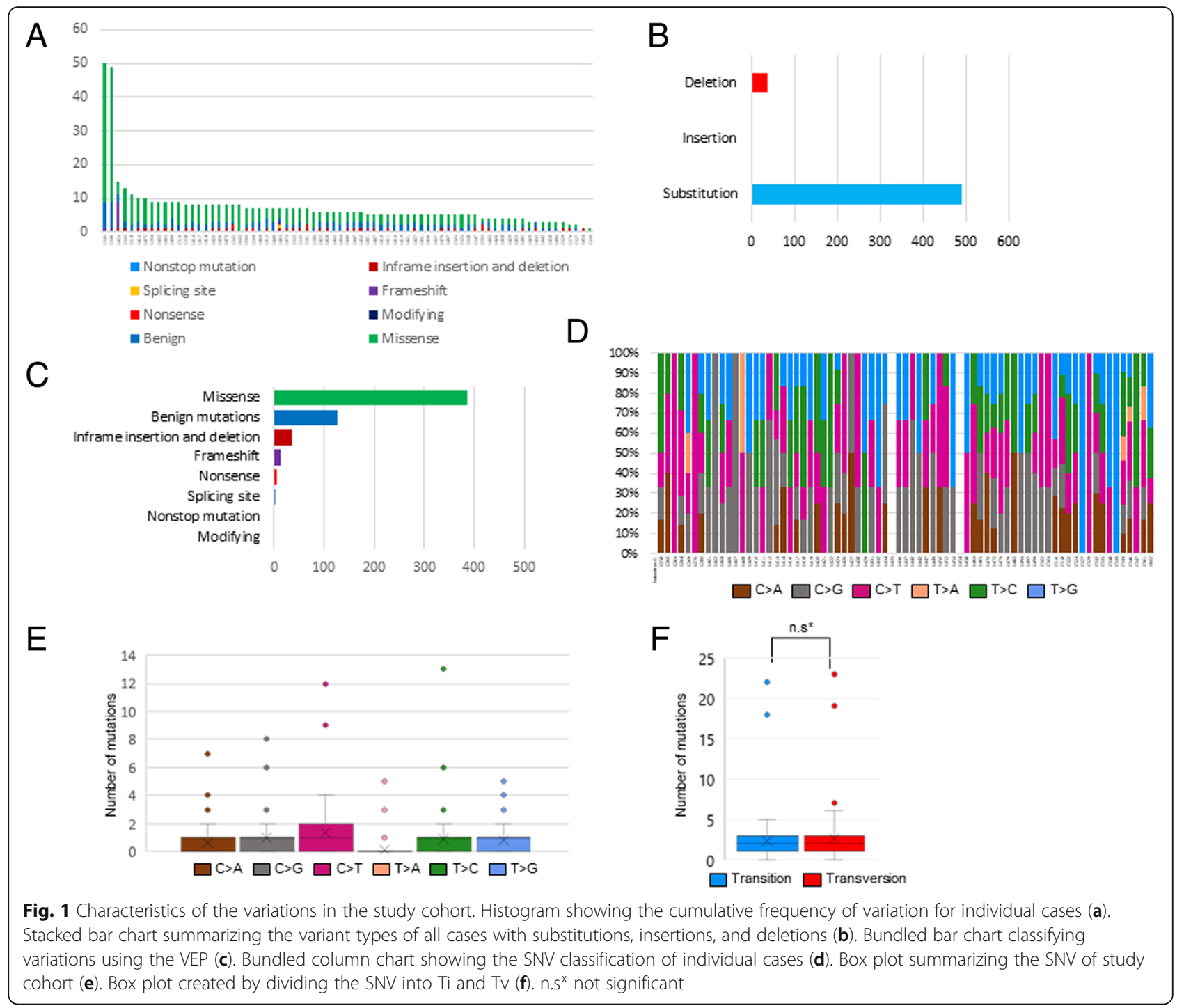

Table 2 Classification of variants in study cohort according to the VEP

\begin{tabular}{ll}
\hline Type of variants & Number of mutations \\
\hline Missense & 346 \\
Benign mutations & 127 \\
Modifying & 0 \\
Nonsense & 6 \\
Frameshift & 13 \\
Splicing site & 1 \\
Inframe insertion and deletion & 37 \\
Nonstop mutation & 0 \\
Total & 530 \\
\hline
\end{tabular}

females, and two cases in which the gender was not reported (Table 1). Of these, 19 had a smoking history. A total of 5272 mutations were recovered from 47 EGFR-TKI-sensitive mutation-positive TCGA-LUAD cases. The median number of total mutations obtained from each sample was 78 [56 112.5], which indicates that the mutational burden was significantly lower than that of other cancer types listed in the TCGA dataset and the TCGA-LUAD parent group (Fig. 3A and B). These 5272 mutations are classified into 4954 single nucleotide substitutions, 146 insertions, and 172 deletions (Fig. 3C). These mutations were further classified according to the VEP and the results are shown in the Table 3 and Fig. 3D.

\section{C > T transition is the most common type of SNV}

Because $\mathrm{C}>\mathrm{A}$ transversion, which is attributed to cigarette smoking, is the most common variation in 


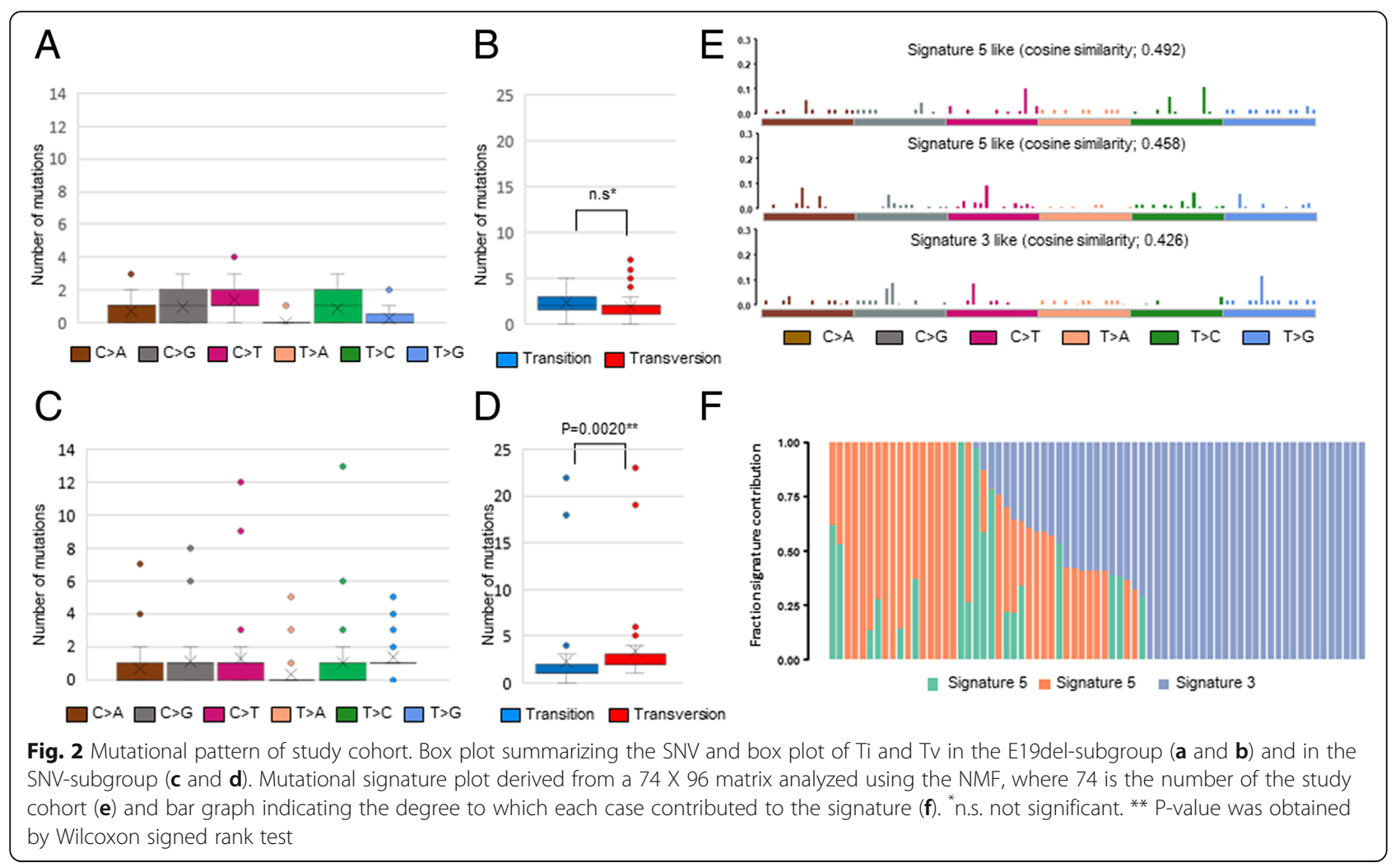

NSCLC [5], we questioned whether this finding also applies to this subset of NSCLC. 1179 mutations, including 1071 synonymous mutations, 47 mutations in the splice region, and 1 stop retaining mutation, were assumed to be harmless or unlikely to change protein behaviors by the VEP; these SNVs along with insertions and deletions were excluded, and 3812 SNVs were classified and analyzed (Fig. 3E and F). In this subset, $\mathrm{C}>\mathrm{T}$ transition accounted for the largest portion (32.7\%), followed by $\mathrm{C}>\mathrm{A}$ transversion (30.3\%). There was no significant difference in the frequency of $\mathrm{C}>\mathrm{T}$ vs. $\mathrm{C}>\mathrm{A}$ mutations in each individual, which contradicts the general notion that NSCLCs harbor higher $\mathrm{C}>\mathrm{A}$ transversions than any other SNV. The difference in the frequencies of $\mathrm{Ti}$ and $\mathrm{Tv}$ in each individual case was not statistically significant (Fig. 3G).

\section{Different mutational spectra between the E19del and SNV-subgroups}

The differences in the mutational pattern observed in the study cohort were further verified in the LUAD cohort. The mutational burden did not differ between the groups with and without a smoking history. In addition, there were no differences in the $\mathrm{Ti}, \mathrm{Tv}$, and $\mathrm{Ti} / \mathrm{Tv}$ ratio according to smoking history or between smokers and non-smokers. There was also no significant difference in the total mutational burden or the frequency of $\mathrm{Ti}$, Tv, and $\mathrm{Ti} / \mathrm{Tv}$ ratio between genders. Finally, we questioned whether the subtypes of EGFR-TKI-sensitizing mutations were related to the difference in mutational burden, $\mathrm{Ti}, \mathrm{Tv}$, and $\mathrm{Ti} / \mathrm{Tv}$ ratio. As seen in the study cohort, there was no statistically significant difference in the frequency of $\mathrm{Ti}$ and $\mathrm{Tv}$ in each individual in the E19del-subgroup, whereas the frequency of Tv was significantly higher than $\mathrm{Ti}$ in the mEGFR SNV-subgroup $(P=0.0220$, Wilcoxon signed rank test). In the E19del-subgroup, the total mutation burden and the number of Tv mutations were significantly lower than in the SNV-subgroup ( $p=0.0497$ and $=0.0220$, respectively, Mann-Whitney $\mathrm{U}$ test) and the $\mathrm{Ti} / \mathrm{Tv}$ ratio was significantly higher as well $(P=0.0055$, Mann-Whitney $U$ test $)$ (Fig. 4A and B).

\section{Heterogeneity of mutational signatures between TCGA- LUAD cases with EGFR-TKI-sensitizing mutations}

Next, in order to estimate the mutagenic stress that influenced EGFR mutations, we analyzed the LUAD cohort using the mutational signature proposed by Alexandrov et al. (4). When inferring the mutagenic stress using NMF, signature 4 (cosine similarity $=0.851$ ), signature 30 (cosine similarity $=0.775$ ), and signature 5 (cosine similarity $=0.713$ ) were derived (Fig. $4 \mathrm{C}$ and D). Because the subset analysis of LUAD cohort according to smoking history, gender, and EGFR mutation subtype showed that only the subtype influenced the distribution of mutational burden, $\mathrm{Ti}$, and $\mathrm{Tv}$, we compared the 


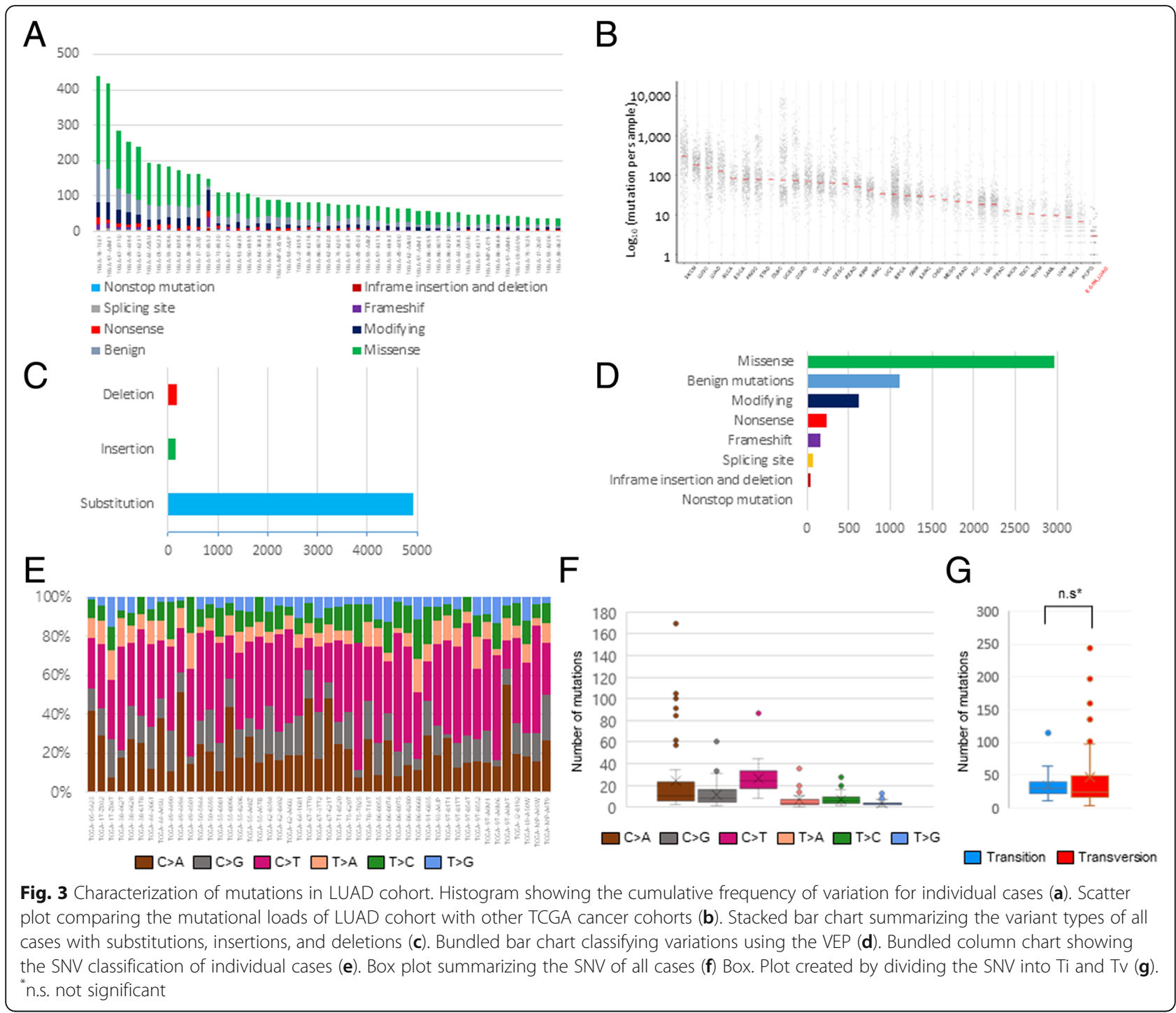

Table 3 Classification of variants in LUAD cohort according to the VEP

\begin{tabular}{ll}
\hline Type of variant & Number of mutations \\
\hline Missense & 2986 \\
Benign mutations & 1127 \\
Modifying & 629 \\
Nonsense & 243 \\
Frameshift & 163 \\
Splicing site & 74 \\
Inframe insertion and deletion & 48 \\
Nonstop mutation & 2 \\
Total & 5272 \\
\hline
\end{tabular}

mutational signatures between the E19del-subgroup and the SNV-subgroup. In the E19del-subgroup, signature 30 (cosine similarity $=0.741$, unknown etiology) and signature 5 (cosine similarity $=0.725$ and $=0.660$, unknown etiology) were derived, whereas signature 4 (cosine similarity $=0.842$, smoking effect), signature 30 (cosine similarity $=0.789$, unknown etiology) and signature 5 (cosine similarity $=0.738$ unknown etiology) were obtained from the SNV-subgroup (Fig. 4E and F). Taken together, the mutational signatures differ between the E19del-subgroup and the SNV-subgroup, as smoking related signature 4 was observed only in SNV-subgroup, and signature 5 and 30 are the prevalent underlying mutational signature in lung adenocarcinoma with mEGFR-TKI.

\section{Discussion}

mEGFR-positive lung adenocarcinoma is a distinctive subtype of lung cancer which attracts attention because 


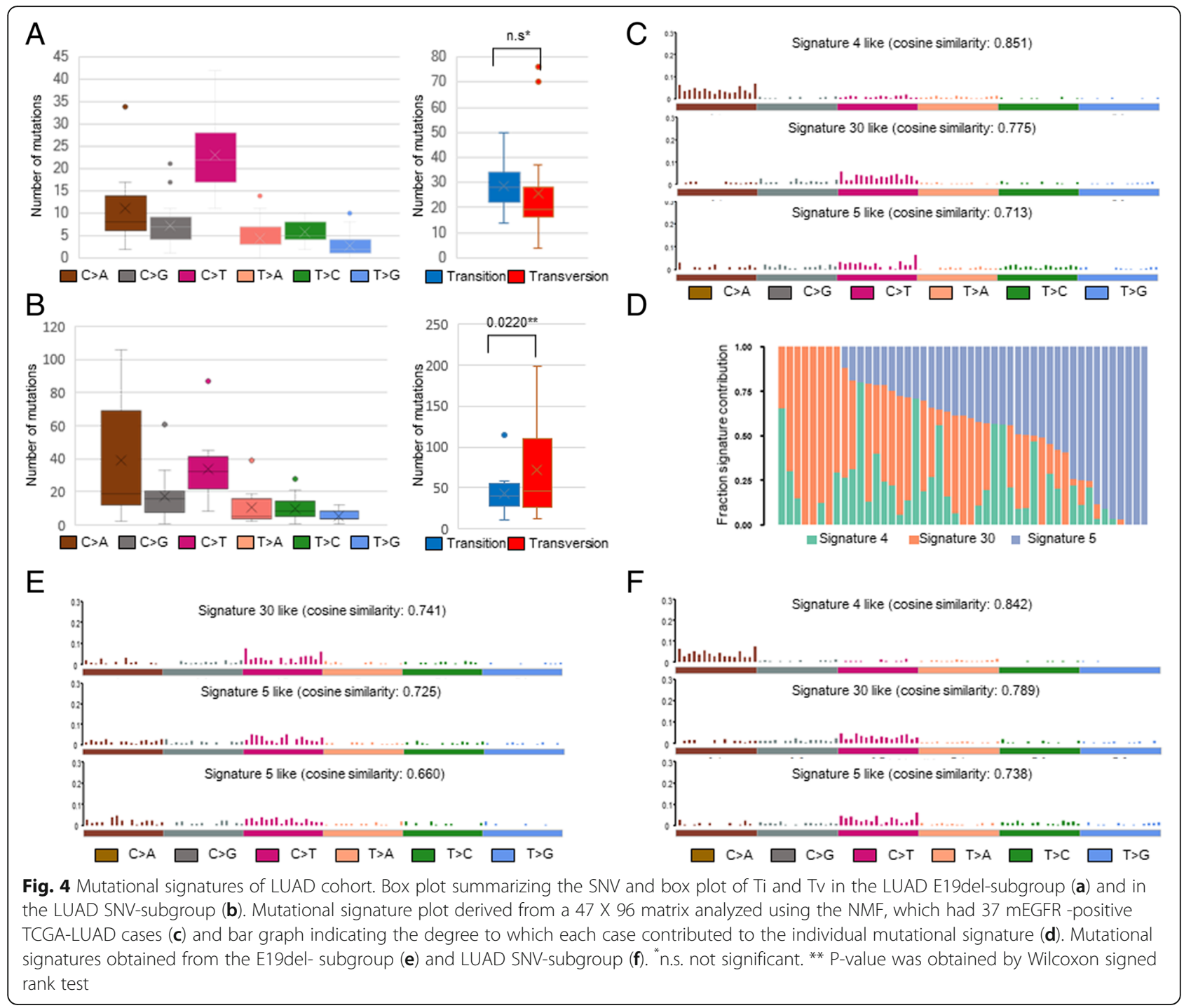

it is a prevalent disease which accounts for about half of East Asian lung adenocarcinomas and because of the facts that it does not involve the typical risk factors for lung cancer such as age, gender, and smoking [19].

The proportion of $\mathrm{C}>\mathrm{A}$ transversions, which is related to tobacco smoking, was not the major SNV, whereas $\mathrm{C}>\mathrm{T}$ transitions comprise the highest proportions of mutations in both cohorts. Another interesting finding is that the proportion of $\mathrm{C}>\mathrm{A}$ transversions is relatively high in the LUAD cohort than study cohort, which may be explained by the fact that the cases in the LUAD cohort had a higher smoking history than that of the study cohort (LAUD cohort: $40.4 \%$ vs. study cohort: $27.8 \%$ ). Further analysis of LUAD cohort according to EGFR subtype revealed that the $\mathrm{Tv}$ frequency was relatively higher, and mutation signature 4 was observed in the SNV-subgroup. Although signature 4 was not derived in the study cohort, it is presumed that mutagenic stressors such as smoking are related to the L858R mutation because the Tv frequency is higher, similar to the LUAD cohort. The E19del-subgroup had lower mutational burden than the SNV subgroup. Both the data from the targeted panel of this study and whole exome data from TCGA-LUAD showed that the mutations of E19del subgroup randomly distributed throughout the genome and the obvious causes could not be detected in the demographic characteristics of E19del subgroup. Younger age in the study cohort and less smoking history in LUAD cohort subgroup might be attributed to these findings. Mutational signature 5 was main variation pattern in the study cohort, whereas signatures 4,30 , and 5 were derived from the analysis of the LUAD cohort. These differences may be attributed to factors such as the higher proportion of smokers in the EGFR-L858R group and higher age in the LUAD cohort compared to the study cohort (LUAD cohort: $66.30 \pm 9.54$ vs. study 
cohort: $60.51 \pm 10.31$ years), and unidentified racial differences. However, during additional analysis by subgroups, signature 5 was predominant and commonly derived, indicating that this maybe one of the key mutational signatures in this cancer type.

The underlying mutational mechanism for signature 5, which exhibits a transcriptional strand bias for $\mathrm{T}>\mathrm{C}$ substitutions at ApTpN context, is yet to be well elucidated. This signature is common in papillary cell renal carcinoma, neuroblastoma, and clear cell renal carcinoma and, in some cancer types, is associated with increased age. However, the correlation between signature 5 and increased age was not observed in the analysis of whole exome sequencing of lung cancer, and it was observed even when we examined the demographic characteristics of our lung cancer set [20]. Among the cancers arising in the kidney, this mutation is characteristic of clear cell and papillary renal cell carcinoma, which absorbs metabolites continuously, whereas it is low in chromophobe renal carcinoma in cortical collecting ducts, suggesting that it may be attributable to the replication error of deaminated cytosine and adenine [21]. Indeed, relentless efforts are required to find out mutagenic stressors other than smoking, such as radon, indoor emissions from household combustion, and exhaust from diesel engines, by collecting the cases enriched with signature 5 and investigating them in various aspects. In contrast, the mutational signature 30, found in a small subset of breast cancers, was observed in the analysis of LUAD cohort; the cause of this mutation pattern has yet to be estimated.

To find out recurrent mutations in specific genes according to the mEGFR subtypes, concurrent mutation was detected by oncoplot and then detected mutations were further examined using the oncodrive function in maftools package, which based on algorithm oncodriveCLUST [22]. In this inspection, the E19del subgroup of study cohort had concurrent mutations in the following order; TP53 > IDH2 $>$ FBXW7 and in the SNV subgroup; TP53> FBXW7 > KRAS. On the other hands, E19del subgroup of LUAD cohort has concurrent mutations as following order; CDKN2A > CEP76 > KIAA2026 and SNV subgroup; AP3D1 > EMR1 > FASTKD3. The mutations observed here were randomly distributed on the genes, and other recurrent driver mutations except mEGFR were not derived.

Targeted sequencing using the Foundation One panel could reflect the results of whole exome sequencing, in terms of the mutational burden [23]. This study was carried out based on this assumption, however, the analysis of study cohort using a customized panel containing 70 major genes covering $0.62 \mathrm{Mb}$ is concerning in terms of direct comparison with the LUAD cohort, which is based on whole exome sequencing. In the future, if the cost is further reduced, it may be necessary to find a minimum sequencing area that can represent whole exome sequencing.

Taken together, the subtype of lung adenocarcinoma with EGFR-TKI-sensitizing mutations does not show a characteristic mutation pattern influenced by smoking and additionally shows a low incidence of $\mathrm{C}>\mathrm{A}$ transversion, which is a common feature of lung cancer; it also had a mutational burden lower than those of other TCGA cancers. E19del and L858R, which are representative subtypes of lung adenocarcinoma, differ in the characteristics of mutations, as the E19del group has a lower mutation burden and a higher ratio of transition than the transversion mutations. Overall, the presence of mutational signatures 5 and 30 was a predominant pattern observed across the subtypes, but the main factors related to this type of signature are still unknown, so they require further in-depth studies on signature 5 and 30 in this particular subtype of lung cancer.

\section{Conclusions}

Lung adenocarcinoma with $\operatorname{mEGFR}(+)$ has a lower mutational burden and does not show a characteristic mutation pattern influenced by smoking. E19del and L858R, which are representative subtypes of mEGFR(+) lung adenocarcinoma, differ in terms of mutational spectra, as the E19del group has a lower mutation burden and a higher $\mathrm{Ti} / \mathrm{Tv}$ ratio. These findings could explain on the differences in the responses to EGFR-TKIs and clinical courses between the two lung adenocarcinoma subgroups.

\section{Additional files}

Additional file 1: Table S1. Type of EGFR mutations in TCGA-LUAD. (DOCX $15 \mathrm{~kb}$ )

Additional file 2: Table S2. List of mEGFR included in this study. (DOCX $12 \mathrm{~kb}$ )

Additional file 3: Table S3. List of TCGA-LUAD cases recruited for this study (LUAD cohort). (DOCX $14 \mathrm{~kb}$ )

Additional file 4: Table S4. List of genes included in the customized NGS panel. (DOCX $12 \mathrm{~kb}$ )

Additional file 5: Table S5. List of study cohort recruited for this study (study cohort). (DOCX $17 \mathrm{~kb}$ )

\section{Abbreviations \\ EGFR: Epidermal growth factor receptor; mEGFR: EGFR-TKI sensitizing mutation; NGS: Next-generation sequencing; NSCLC: non-small cell lung cancer; SNV: single nucleotide variation; Ti: transition; TKI: tyrosine kinase inhibitor; TV: transversion; VEP: variant effect predictor}

\section{Acknowledgements}

Not applicable.

Funding

This work was supported by the National Research Foundation of Korea (NRF) grant awarded to YS Chang by the Korea government (MSIT) (No. NRF2017R1A2B4009017). The funding body did not play any role in the design of the study, and collection, analysis, and interpretation of data and in writing the manuscript. 


\section{Availability of data and materials}

All reagents used in this study are commercially available from the listed companies. FFPE tissue blocks can be obtained from the tissue registries of Severance and Gangnam Severance Hospital, which are affiliated hospitals of Yonsei University College of Medicine and can be obtained after IRB evaluation and approval. The datasets used and analyzed during the current study are available from the corresponding author on reasonable request.

\section{Authors' contributions}

EYK participated in the design of the study, carried out the IRB process, performed experiments, and wrote the manuscript draft. AK performed the experiment and generated data. GEL was responsible for statistical analysis of the data and $\mathrm{HL}$ participated in the conception and design of the study and contributed to the analysis and interpretation of the data. YSC conceived the study and also participated in its design and coordination and helped to draft the manuscripts. All authors read and approved the final manuscript.

\section{Ethics approval and consent to participate}

This study involves human derived material and data and was carried out in compliance with the declaration of Helsinki (https://www.wma.net/policiespost/wma-declaration-of-helsinki-ethical-principles-for-medical-researchinvolving-human-subjects/) and Korean GCP guidelines. This study was approved by the IRB of Gangnam Severance Hospital (IRB \#3-2017-0059) and written informed consents were obtained from participants on the use of human derivatives.

\section{Consent for publication}

The verbal consent that the data obtained from the specimens might be published anonymously were obtained from the human derivative providers.

\section{Competing interests}

Every author declared that there is no conflict of interest.

\section{Publisher's Note}

Springer Nature remains neutral with regard to jurisdictional claims in published maps and institutional affiliations.

\section{Author details}

${ }^{1}$ Department of Internal Medicine, Yonsei University College of Medicine, 50-1 Yonsei-ro, Seodaemun-gu, Seoul, Republic of Korea. ${ }^{2}$ Department of Mathematics, Sungkyunkwan University, 25-2 Sungkyunkwan-ro, Jongno-gu, Seoul, Republic of Korea. ${ }^{3}$ Department of Internal Medicine, Yonsei Univeristy College of Medicine, 4th Floor Research Center for Future Medicine, 63-Gil 20, Eonju-ro, Gangnam-gu, Seoul, Republic of Korea.

Received: 28 May 2018 Accepted: 20 November 2018 Published online: 06 December 2018

\section{References}

1. Torre LA, Bray F, Siegel RL, et al. Global cancer statistics, 2012. CA Cancer J Clin. 2015;65:87-108

2. Jung K-W, Won Y-J, Oh C-M, et al. Cancer statistics in Korea: incidence, mortality, survival, and prevalence in 2014. Cancer Res Treat. 2017;49: 292-305.

3. Ferlay J, Soerjomataram I, Dikshit R, et al. Cancer incidence and mortality worldwide: sources, methods and major patterns in GLOBOCAN 2012. Int Cancer. 2015;136:E359-86.

4. Pearce $N$, Blair A, Vineis $P$, et al. IARC monographs: 40 years of evaluating carcinogenic hazards to humans. Environ Health Perspect. 2015;123:507-14.

5. Alexandrov LB, Ju YS, Haase K, et al. Mutational signatures associated with tobacco smoking in human cancer. Science. 2016:354:618-22.

6. Stratton MR, Campbell PJ, Futreal PA. The cancer genome. Nature. 2009:458:719

7. Pfeifer GP. Environmental exposures and mutational patterns of cancer genomes. Genome med. 2010;2:54.

8. Alexandrov LB, Nik-Zainal S, Wedge DC, et al. Signatures of mutational processes in human cancer. Nature. 2013;500:415-21.

9. Helleday T, Eshtad S, Nik-Zainal S. Mechanisms underlying mutational signatures in human cancers. Nat Rev Genet. 2014;15:585-98.

10. Alexandrov LB, Nik-Zainal S, Wedge DC, et al. Deciphering signatures of mutational processes operative in human Cancer. Cell Rep. 3:246-59.
11. Nik-Zainal S, Alexandrov Ludmil B, Wedge David C, et al. Mutational processes molding the genomes of 21 breast cancers. Cell. 2012;149:979-93.

12. Midha A, Dearden S. McCormack R. EGFR mutation incidence in non-smallcell lung cancer of adenocarcinoma histology: a systematic review and global map by ethnicity (mutMapll). Am J Cancer Res. 2015;5:2892-911.

13. Pirker R, Herth FJ, Kerr KM, et al. Consensus for EGFR mutation testing in non-small cell lung cancer: results from a European workshop. J Thorac Oncol. 2010;5:1706-13.

14. Mok TS, Wu Y-L, Thongprasert S, et al. Gefitinib or carboplatin-paclitaxel in pulmonary adenocarcinoma. New Engl J Med. 2009;361:947-57.

15. Chang YS, Choi CM, Lee JC. Mechanisms of epidermal growth factor receptor tyrosine kinase inhibitor resistance and strategies to overcome resistance in lung adenocarcinoma. Tuberc Respir Dis (Seoul). 2016;79:248-56.

16. Park K, Tan EH, O'Byrne K, et al. Afatinib versus gefitinib as first-line treatment of patients with EGFR mutation-positive non-small-cell lung cancer (LUX-lung 7): a phase 2B, open-label, randomised controlled trial. Lancet Oncol. 2016:17:577-89.

17. Alexandrov LB, Stratton MR. Mutational signatures: the patterns of somatic mutations hidden in cancer genomes. Curr Opin Genet Dev. 2014:24:52-60.

18. Mayakonda A, Koeffler HP. Maftools: Efficient analysis, visualization and summarization of MAF files from large-scale cohort based cancer studies. bioRxiv 2016.

19. Kim ES, Hirsh V, Mok T, et al. Gefitinib versus docetaxel in previously treated non-small-cell lung cancer (INTEREST): a randomised phase III trial. Lancet. 2008;372:1809-18.

20. Alexandrov LB, Jones $\mathrm{PH}$, Wedge DC, et al. Clock-like mutational processes in human somatic cells. Nat Genet. 2015;47:1402-7.

21. Davis CF, Ricketts CJ, Wang M, et al. The somatic genomic landscape of chromophobe renal cell carcinoma. Cancer Cell. 2014;26:319-30.

22. Tamborero D, Gonzalez-Perez A, Lopez-Bigas N. OncodriveCLUST: exploiting the positional clustering of somatic mutations to identify cancer genes. Bioinformatics. 2013;29:2238-44.

23. Campbell BB, Light N, Fabrizio D, et al. Comprehensive analysis of Hypermutation in human Cancer. Cell. 2017;171:1042-56

\section{Ready to submit your research? Choose BMC and benefit from:}

- fast, convenient online submission

- thorough peer review by experienced researchers in your field

- rapid publication on acceptance

- support for research data, including large and complex data types

- gold Open Access which fosters wider collaboration and increased citations

- maximum visibility for your research: over $100 \mathrm{M}$ website views per year

At BMC, research is always in progress.

Learn more biomedcentral.com/submissions 